\title{
Communication Skills Improving Assistance
}

\author{
Tejas Sayankar, Insiyah Kanchwala, Nikita Mahajan, Sayali Mahajan \\ Department of Computer Engineering, MET's Institute of Engineering, Nashik, Maharashtra, India
}

\begin{abstract}
How to cite this paper: Tejas Sayankar | Insiyah Kanchwala | Nikita Mahajan | Sayali Mahajan "Communication Skills Improving Assistance" Published in International Journal of Trend in Scientific Research and Development (ijtsrd), ISSN: 24566470, Volume-3 | Issue-4, June 2019, pp.568-570, URL: https://www.ijtsrd. com/papers/ijtsrd2 3748.pdf

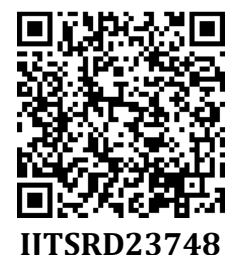

Copyright (c) 2019 by author(s) and International Journal of Trend in Scientific Research and Development Journal. This is an Open Access article distributed under the terms of the Creative Commons

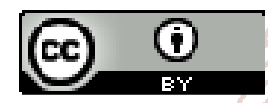
Attribution License (CC BY 4.0) (http://creativecommons.org/licenses/ by $/ 4.0$ )

\section{Introduction}

This section describes the term Virtual Reality and introduces the concept of Virtual Reality Technology. It also gives the overview of the system which describes the deliverables of the project.

\subsection{Overview}

Virtual Reality is a computer technology that generates realistic images, sounds and other sensations that simulate a user's physical presence in a virtual environment. The proposed system allows a person to face a situation where he/she must speak staying in a simulated world with no fear. This type of immersive learning in the can provide learners a real opportunity to learn by doing, thereby increasing motivation and retention.

\section{Literature Survey}

Despite recent technological advances that make VR systems more appealing to Clinical practice, there is still a considerable lack of scientific evidence of how VR systems can be meaningfully implemented in existing rehabilitation routines. A number of recent reviews have been published on the use of VR and video games for physical rehabilitation. The reviewed studies conclude that VR technology allows therapy to be provided within a functional, purposeful and motivating context V-Buddy is an audio-based application, is designed to provide a solution to overcome students' lack of confidence to speak in Virtual World. This interdisciplinary study Integrates ICT in education through the innovation of an interactive audio-based application as a tool to enhance
English language speaking skills among less proficient Students. Drawing on the socio cultural perspective of learning, the application named 'V-Buddy' has been developed and tested with a group of participants which consists of five primary school students and an English language teacher. Adopting one group pre-test and post-test experimental design as its methodology, the teacher was asked to evaluate the students' level of confidence to speak prior to and after their engagement with the V-Buddy. Cambly gives instant access to native English speakers over video chat. Complete training is given by professional trainers through a video call. Cambly is an online tutoring platform that connects English learners in more than 130 countries with native speakers. Cambly offers English language tutoring services 24/7. The company inactively seeking dynamic, engaging and patient Native English speakers from around the world to work for them as online tutors. When you talk to a student or paying Cambly user, you get paid to speak conversationally with the student and provide any help that is needed by the student while you both are online. If you are a native English speaker, this should be pretty easy. Unreal Engine 4 is a suite of integrated tools for game developers to design and build games, simulations, and visualizations. One virtual reality games was researched and developed, based on Unreal engine 4 and the HTC Vive. Some key technologies was used in this game, such as path finding through algorithm or waypoints, particle system, file access and other technologies, combined with 3D sound effect and UI editor, etc. Autodesk 3ds Max is a professional 3D computer graphics program for making 3D 
animations, models, games and images. The proposed system helps an individual to improve himself with suggestions provided by trainers/Judges. The learner is placed in such situation where he can see people in front but in actual that crowd is just a simulated character. Autodesk's 3Ds Max Interactive helps produce visually stunning, immersive, and interactive experiences of every kind.

\section{Problem Definition}

Students are not able to present themselves in crowd of people, and this has become an existing problem these days. So with help of survey we decided to address the solution and to develop a VR based project to nourish ones communication skills. With the unique combination of online training and virtual reality, a person will be able to improve his/her public speaking skills in a safe environment and overcome any speech anxiety. As virtual reality provides a realistic environment practicing in such Environment will result in upgrading ones communication skills. Regular practicing will lead to overcome once stage fear or phobia of stage fear and will be able to present themselves in front of crowd.

\subsection{Need of System}

Virtual Reality is a technology which is widely used in many areas and here in this system, motive is to build person's skills by providing realistic environment and regular evaluation for individual improvement.

\subsection{Basic Idea}

To overcome the realistic issues faced by people in today's world led us to build this system which targets in helping people to overcome drawbacks in them. UsingVR technology we are trying to develop a software that provides realistic environment in which student has to speak and audio video is been recorded and sent to admin/trainer for evaluation sake. Depending on result improvement of student is judged.

\section{Analysis}

All possible requirements of the system to be developed are captured in this phase and documented in a requirement specification document. We have gathered requirement and also gone through a survey which led us to the existing systems which are already developed. After comparison among all, we decided features of our system.

\section{Design}

The requirement specifications from first phase are studied in this phase and the system design is prepared. This system design helps in specifying hardware and system requirements and helps in defining the overall system architecture. In this phase, we have designed the basic work ow of system in terms of Data Flow Diagrams and UML Diagrams.

\subsection{Software Requirement Specifications}

The Software Requirement Specification describes the scope of the project, operating environment, user characteristics, design and constraints. It also elaborates the system architecture of the software.

\subsubsection{Project Scope}

The world is acknowledging and accepting the concept of Virtual Reality but the scope and the application of the technology is unexplored and unknown by many. Imagine taking lectures in a hall and you sitting in your room, or watching the game of your favorite sport from your home. All this is now possible and achievable with Virtual Reality. VR is the magic born from the womb of technology which has tantalizing possibilities and promises for a future bright and smart. VR was initially only confined for gaming, but it is already being put to any innovative and brilliant uses. The proposed system cures fear of Public speaking. People are trained by themselves for speaking in public through virtual reality. Many people all around the world suffer from stage freight and have never been up on the stage or any public platform due to this fear. It could be easily overcome through the use of VR and the system which is under development.

\subsubsection{Operating Environment}

It is very important that environment must be realistic, so that the users can experience that they are in real environment which will help them to practice. For that we have to create realistic 3D objects with proper texturing. Environment lightning should be proper so that it becomes more realistic. We are creating environments in Unreal Engine4.After creation of environment model are going to add some proper lightning to it. By this it will look photorealistic.

\subsubsection{User Classes and Characteristics}

The user who is going to operate the system should have the good Internet connection to control remotely the application.

\subsection{System Architecture}

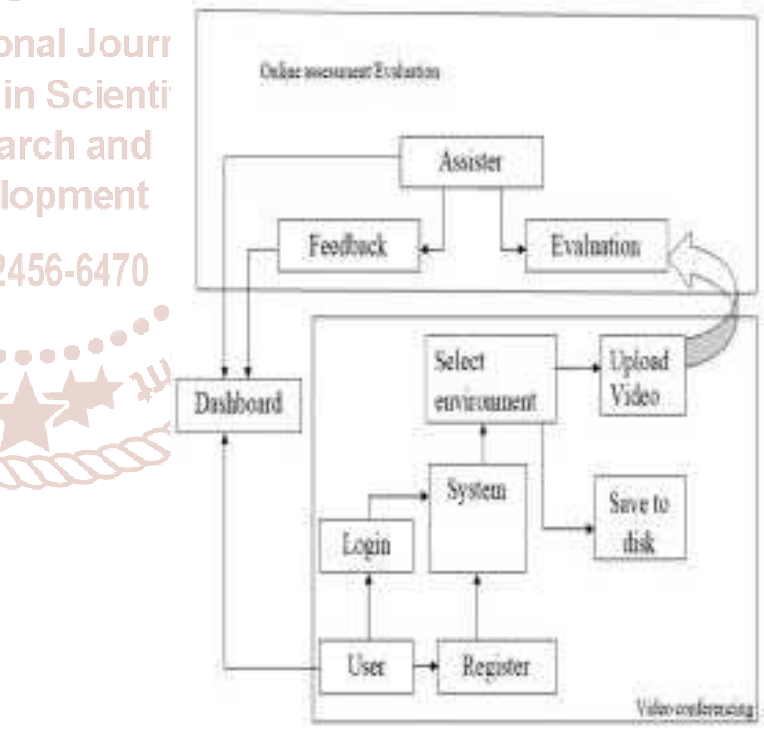

Fig .5.2.System Architecture

\section{Modeling}

This section includes the modeling technique which describes the system. It also describes the functionality of the different features of the technology been used.

\subsection{Class Diagram}

The class diagram shows the building blocks of any objectoriented system. Class diagram depicts a static view of the model or part of the model, describing what attributes and behavior it has rather that the detailing the methods of achieving operations. Class diagrams are most useful in illustrating relationships between classes and interfaces. Generalizations, aggregations and associations are all valuable in reacting interface, composition or usage and connections receptively. 


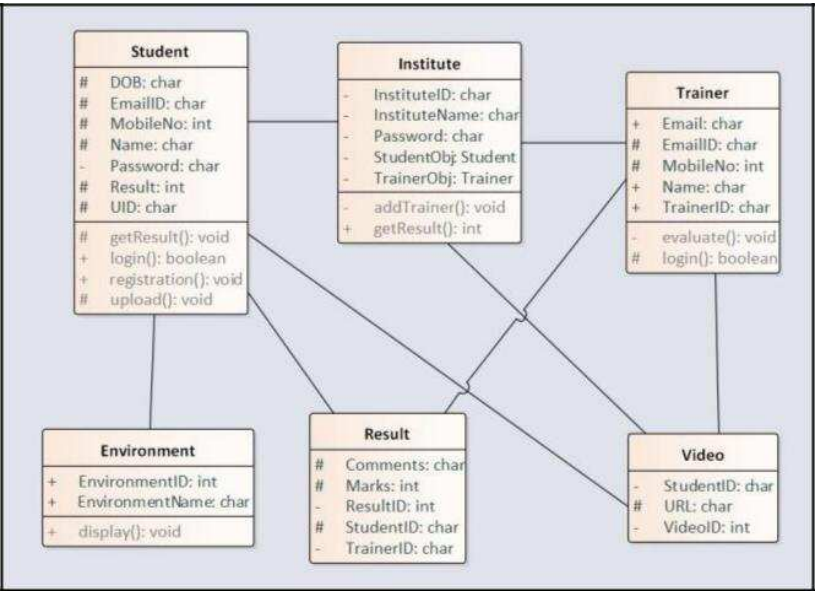

Fig. 6.1 Class Diagram

\section{Implementation Details}

\subsection{Android Application}

Basic steps we followed to create Android Application for VR technology are:

1. Asset creation: First we modelled all the $3 \mathrm{~d}$ objects required in the environment. For that, we used Autodesk $3 \mathrm{~d}$ Max. After modelling 3d objects, we applied textures on those objects.

2. Environment creation: Then we created an environment in Unreal Engine 4. We applied proper lighting on it to give a realistic look. Here we import the entire asset which is required to place in an environment such as tables, chairs, characters, etc. and placed it in a proper position.

3. Enable plug-in: As we have to develop application for cardboard VR, we enabled Google VR plug-in before packaging the project.

4. Setup for packaging: We applied all the required project setting for a controller, input, default game mode, default level, etc. We also created a main menu widget.

5. Packaging: Then we start packaging the project for android platform.

\subsection{Web Application}

As the system functions to evaluate video of student performed in particular environment. So web application provides platform for trainer and student to register themselves and upload the video and evaluate respectively. The application generates result which is viewed by student and also comments if any by the trainer.

\section{Technical Specifications}

\subsection{Advantages}

1. Improves communication of people on the basis of regular evaluation and reduces chances of Jargons while speaking.

2. User will be self-confident to present himself/herself.

3. Overcomes phobia of speaking in front of bulk of people.

4. Creates Interest in person to learn.

5. Evaluation will be done easily on the basis of specified criteria like language Fluency, content of speech, gestures, eye contact, speech delivery, voice tone, etc.

\subsection{Limitations}

1. Limited for Extempore situation (Other situations like Group Discussion, HR Interviews, etc. are not supported).

\subsection{Applications}

1. It provides a virtual environment in which students can safely practice real-world skills.

2. Helps in distance learning.

\subsection{Hardware and Software Requirement \\ 8.4.1 Hardware Requirement}

Hardware and software requirements for the system are stated below:

1. Google cardboard for virtual experience.

2. Computer system with Webcam and internet connection.

3. Android smartphone with Gyro sensor.

\subsubsection{Software Requirements}

Unreal engine4

Autodesk 3ds max

Wamp Server

\section{Conclusion}

We are developing a VR based (verbal/non-verbal) interactive system for developing communication skills based on predefined and planned of greeting scenario. Our project consists of 2 modules. First module is for Evaluation and second module is for Realistic Environment. To create realistic environment, we are using Unreal Engine 4, Because it supports photo-realistic environments. We are using Autodesk 3ds Max for modelling 3d objects. It is easy to use than other software's like Autodesk Maya or Blender or Cinema 4D. The system provides various environments nbased on levels which are been faced by the users. The result of practice test will lead to improvement of speaking in front of audience as well as development of individual's personality. Difficulties raised in each environment will result inup-gradation of selfdom.

\section{Reference}

[1] Max Ortiz-Catalan, Sharon Nijenhuis and Kurt Ambrosch, \Virtual Reality", http://ict.usc.edu/pubs/Virtual Reality.pdf.

[2] Radzuwan Ab Rashid and S. B. Mohamed, \Developing Speaking Skills Using Virtual Speaking Buddy", in International Journal of Emerging Technologies in Learning (iJET), May 2017.

[3] Cambly, \https://www.cambly.com/englis h?lang=en"

[4] Xiaowei Chen, Meihong Wang and Qingfeng $\mathrm{Wu}$, $\backslash$ Research and development of virtual reality game based on unreal engine 4", in 2017 4th International Conference on Systems and Informatics (ICSAI).

[5] Autodesk 3ds Max, https://www.autodesk.in/products/3dsmax/overview" 